\title{
EL MODELO DE SEGURIDAD ALIMENTARIA DE LA UNIÓN EUROPEA Y SU DIMENSIÓN EXTERIOR
}

\author{
Mercedes Guinea ${ }^{1}$ \\ Universidad Complutense de Madrid (UCM)
}

\begin{abstract}
Resumen:
La Unión Europea, tercer grupo humano del planeta, es el primer importador mundial y segundo exportador de productos alimenticios. Es un actor global en materia de seguridad alimentaria, cuyas decisiones afectan sustancialmente al comercio mundial de alimentos y, por tanto, a las políticas alimentarias del resto de los Estados, especialmente, de aquellos que necesitan del comercio para asegurar una oferta suficiente de alimentos para su población. En este trabajo nos proponemos examinar el modelo de seguridad alimentaria de la UE, regulado a través de la PAC, y la dimensión exterior de este modelo, esto es, las relaciones comerciales sobre productos agrícolas que la UE mantiene con sus socios así como la cooperación para el desarrollo. Esto es relevante para los países del Magreb, ya que la UE determina con sus políticas internas tanto la disponibilidad de alimentos como los precios de los mercados internacionales. Tiene que ser un referente para el resto de los países de su entorno y, especialmente, con aquellos con los que tiene relaciones comerciales específicas en materia de comercio de alimentos.
\end{abstract}

Palabras clave: Unión Europea, Política Agrícola Común (PAC), política comercial en materia agrícola, cooperación para el desarrollo en seguridad alimentaria.

Title in English: "The European Union's Food Security Model and its External Dimension”

\section{Abstract:}

The European Union is the third human group in the planet, the first world food importer and the second food exporter. It is a global actor in food security, whose policies affect world agricultural trade and, consequently, food security policies of every State in the world, especially those dependent on imports for food supplies. In this paper we examine the EU food security model, established by the CAP, and its international aspects, food trade relations and development cooperation. This is important for the Maghreb countries, as European internal policies have an impact on international food availability and international market prizes. EU policies are a referent for its neighbouring countries because of close trade policies and cooperation ties.

Keywords: European Union, Common Agricultural Policy (CAP), agricultural trade policy, food security development cooperation.

Copyright $@$ C UNISCI, 2013.

Las opiniones expresadas en estos artículos son propias de sus autores, y no reflejan necesariamente la opinión de UNISCI. The views expressed in these articles are those of the authors, and do not necessarily reflect the views of UNISCI.

\footnotetext{
${ }^{1}$ Mercedes Guinea Llorente es Profesora de Relaciones Internacionales de la Universidad Complutense de Madrid, titular del Módulo Europeo Jean Monnet de la Comisión Europea sobre "La aplicación del Tratado de Lisboa: efectos en el modelo político, económico y social". Es igualmente investigadora senior de UNISCI y miembro del Foro Hispano-Argelino.

Dirección: Departamento de Estudios Internacionales, Facultad de Ciencias Políticas y Sociología, UCM, Campus de Somosaguas, 28223 Madrid, España.
}

Email: mercedes.guinea@telefonica.net.

http://dx.doi.org/10.5209/rev_UNIS.2013.n31.4470 


\section{Consideraciones generales: el modelo de seguridad alimentaria de la UE}

La Unión Europea, tercer grupo humano del planeta, es el primer importador mundial y segundo exportador de productos alimenticios. Es un actor global en materia de seguridad alimentaria, cuyas decisiones afectan sustancialmente al comercio mundial de alimentos y, por tanto, a las políticas alimentarias del resto de los Estados, especialmente, de aquellos que necesitan del comercio para asegurar una oferta suficiente de alimentos para su población.

Antes de comenzar con el análisis del modelo de seguridad alimentaria europeo, es preciso hacer unas aclaraciones previas. Los conceptos de seguridad alimentaria de la Unión Europea y el de la Organización de Naciones Unidas para la Agricultura y la Alimentación (FAO) difieren entre sí. Para la FAO "existe seguridad alimentaria cuando todas las personas tienen en todo momento acceso físico, social y económico a suficientes alimentos inocuos y nutritivos para satisfacer sus necesidades alimenticias y sus preferencias en cuanto a los alimentos a fin de llevar una vida activa y sana. Los cuatro pilares de la seguridad alimentaria son la disponibilidad, el acceso, la utilización y la estabilidad. La dimensión nutricional es parte integrante del concepto de seguridad alimentaria" ${ }^{2}$.

Por el contrario, para la Unión Europea la seguridad alimentaria sólo afecta al pilar de la salud y seguridad de los consumidores, afectando a la normativa relativa a la higiene de los productos alimenticios, a la salud y bienestar de los animales, a la fito sanidad, a la prevención de los riesgos de contaminación por sustancias externas y al etiquetado adecuado de dichos productos ${ }^{3}$.

Nosotros aplicaremos el concepto de la FAO al estudio de la producción y comercialización de alimentos en la UE, esto es, el modelo de seguridad alimentaria de la UE, pero obviando el enfoque más restrictivo que este concepto tiene en el Derecho Comunitario. El modelo de seguridad alimentaria del conjunto de la UE viene determinado por su Política Agrícola Común (PAC), política única que se aplica a la producción, distribución y comercialización de alimentos en los 27 Estados de la UE, sin que exista la posibilidad de que los Estados miembros adopten políticas propias. La producción y disponibilidad de alimentos también resulta afectada por otra de las políticas comunes, la Política Común de Pesca, que, por razones de espacio y menor importancia para la provisión alimentaria, aquí no vamos a analizar.

En este trabajo nos proponemos examinar el modelo de seguridad alimentaria de la UE, regulado a través de la PAC, y la dimensión exterior de este modelo, esto es, las relaciones comerciales sobre productos agrícolas y alimentarios que la UE mantiene con sus socios. Esto es relevante, a nuestros efectos, por el carácter de la UE de actor principal del comercio internacional de alimentos y, además, por ser el socio comercial de referencia de los países del Magreb, objeto del presente estudio ${ }^{4}$. Por tanto, la UE determina con sus políticas internas tanto la disponibilidad de alimentos como los precios de los mercados internacionales. Tiene

\footnotetext{
${ }^{2}$ Organización de las Naciones Unidas para la Agricultura y la Alimentación (FAO): Declaración de la Cumbre Mundial sobre la Seguridad Alimentaria, WSFS 2009/2, Roma, (16-18 de noviembre de 2009), p. 1.

${ }^{3}$ Sobre la seguridad alimentaria de la UE, puede verse: Costato, Luigi y Albisinni, Ferdinando (eds.) (2012): European Food Law, Roma, CEDAM; McDonald, Brian L. (2010): Food Security, Cambridge, Polity Press; McElhatton, Anna y Marshall, Richard J. (eds.) (2007): Food Safety: A Practical and Case Study Approach, New York, Springer; MacMaoláin, Caoimhim (2007): EU food law : protecting consumers and health in a Common Market, Oxford, Hart; Oanta, Gabriela Alexandra (2007): La política de seguridad alimentaria en la Unión Europea, Valencia, Tirant lo Blanch.

${ }^{4}$ Las relaciones concretas en materia de comercio de alimentos entre la UE y los tres países del Magreb son objeto de estudio en este mismo número por María Ángeles Alaminos y Beatriz Tomé.
} 
que ser un referente para el resto de los países de su entorno y, especialmente, con aquellos con los que tiene relaciones comerciales específicas en materia de comercio de alimentos.

Examinando el modelo de la PAC, y cualquier modelo de política de seguridad alimentaria, nos encontramos con que puede responder a tres modelos diferentes, que podríamos denominar, atendiendo a sus objetivos políticos, como productivismo, neoliberalismo y multifuncionalismo.

- Productivismo: Es el modelo original de la PAC, donde prima la preocupación por la seguridad y autosuficiencia alimentaria europea, considerada desde su dimensión estratégica clave para la seguridad general. Esa autosuficiencia se garantiza incentivando la producción de alimentos en la Comunidad Europea a través del sostenimiento y la garantía de los precios de los alimentos y medidas del mercado comunitario frente al exterior.

- Neoliberalismo: Sería un modelo caracterizado por la aplicación exclusiva de las leyes de oferta y demanda a la producción y comercialización de productos alimentarios, eliminándose cualquier excepcionalidad que estos productos pudieran tener por razón de su carácter estratégico. Es el modelo que trata de imponerse por presiones del comercio internacional, especialmente por la posición de negociación de los Estados emergentes en el seno de la Organización Mundial de Comercio. Las medidas políticas necesarias para imponer este modelo serían la prohibición de la intromisión de cualquier autoridad pública en las condiciones de producción y comercio de alimentos, eliminándose las subvenciones y cualquier otra medida que distorsione el comercio.

- Multifuncionalismo: Este modelo, que es el que actualmente se está desarrollando en el seno de la UE, considera la agricultura como un sector estratégico, que provee a la sociedad de "bienes públicos globales", y no sólo de alimentos. Se protege al agricultor, no sólo por su importancia como productor de alimentos, sino por ser un "agente social" fundamental para la prestación de otros servicios como la preservación medioambiental, el equilibrio demográfico campo-ciudad, la viabilidad económica y social de las zonas rurales, la preservación de culturas y tradiciones locales, etc. Este modelo se articula a través de medidas de sostenimiento de las rentas de los agricultores, independientemente de lo que produzcan, en un entorno de comercio liberalizado.

La reforma de la PAC, propuesta por la Comisión Europea, y todavía en negociación, propone continuar con el multifuncionalismo hacia dentro, para hacer posible un neoliberalismo (matizado y exigido por los compromisos internacionales) hacia fuera. Examinaremos estos aspectos a través de un estudio a lo largo del tiempo de los modelos de seguridad alimentaria de la PAC y de las medidas de comercio internacional y cooperación para el desarrollo que afectan al comercio de los alimentos con terceros Estados.

La tesis que defenderemos es que las tendencias actuales de demanda del contexto internacional así como las presiones del cambio climático aconsejan reconsiderar el modelo de seguridad alimentaria de la PAC. El modelo multifuncionalista actual debería priorizar otra vez la producción de alimentos, que vuelve a ser una urgencia, combinándola con medidas de protección medioambiental. 


\section{El modelo de seguridad alimentaria de la UE: la Política Agrícola Común (PAC)}

La PAC en la actualidad, según la Comisión Europea, busca responder a la demanda pública de un sector agrícola sostenible en Europa a través de reforzar la competitividad del sector agrícola, asegurar una oferta de alimentos suficientes y seguros, preservar el medio ambiente y el entorno rural así como proveer de un nivel de vida justo a la comunidad agrícola ${ }^{5}$. Puede verse así como este modelo responde a las características del multifuncionalismo, en la medida en que se pretende de la PAC que garantice una variedad de objetivos de carácter político, económico y social ${ }^{6}$.

El modelo de seguridad alimentaria de los Estados de la UE se define en el nivel comunitario, sin que los niveles de los Gobiernos nacionales tengan poder de decisión. La política de seguridad alimentaria de la UE se define en base a las necesidades, prioridades y objetivos del conjunto, pero no responde a los intereses de cada uno de los Estados, que sólo pueden intentar defender sus preferencias a través de las distintas instituciones comunitarias. Trata de responder a una visión de conjunto sin que las especificidades nacionales reciban una respuesta individualizada, aun existiendo contextos muy diferentes de unos Estados miembros a otros, ya sea climatológicamente, orograficamente, demográficamente, económicamente o socialmente.

El sector de la producción y comercialización de los productos alimenticios es el único mercado realmente común de la UE, cuya política se define se definen las prioridades políticas y se legisla en el seno de las instituciones europeas, y los Estados miembros sólo aplican y ejecutan esa política, que puede considerarse única ${ }^{7}$. Es una política que afecta a un mercado compuesto por 27 Estados miembros y que engloba a 495 millones de personas, tercer mercado del planeta, sólo después de China y la India. La PAC implica, por tanto, la existencia de reglas únicas de producción, comercialización y distribución de productos alimenticios y de protección de la salud y seguridad de los consumidores y del bienestar de los animales aplicables a ese mercado. Igualmente supone una financiación común de esa política con cargo al presupuesto comunitario, constituyendo todavía al día de hoy una de las principales partidas de gasto 8 .

\footnotetext{
${ }^{5}$ European Commission, Directorate-General for Agriculture and Rural Development: "Why do we need a Common Agricultural Policy?", Agriculture and Rural Development, Discussion Paper DG Brussels, Diciembre 2009, p. 1.

${ }^{6}$ En relación a la PAC pueden consultarse los siguiente trabajos de referencia: Bluman, Claude, Blanquet, Marc et al. (eds.) (2011): Politique agricole commune et politique commune de la pêche, Bruxelles, Université Libre de Bruxelles, Commentaires J. Mégret; Cardwell, Michael (2004): The European Model of Agriculture, Oxford, Oxford University Press; Carretero García, Ana: "La política agraria común”, en: Ortega, Luis y De La Sierra, Susana (dirs.) (2011): Estudios de la Unión Europea, Toledo, Centro de Estudios Europeos, pp. 175-223; Celma Alonso, Pilar (2004): Las claves de la Política Agrícola Común, Madrid, Colex; Del Saz Cordero, Silvia y Celma Alonso, Pilar: "La Política Agrícola Común", en Linde Paniagua, Enrique (ed.) (2008): Políticas de la Unión Europea, Madrid, Colex, pp. 222-269; García Delgado, José Luis y García Grande, Ma Josefa (2005): Política Agraria Común: balance y perspectivas, Barcelona, La Caixa; Grant, Wyn (1997): The Common Agricultural Policy, London, Macmillan; Langmaier, Thomas (2010): Common Agricultural Policy and EU Agriculture: The Effects of the Reforms since 1992, Saarbrücke, Verlag Dr. Müller; Ritson, Christopher y Harvey, David (1997): The Common Agricultural Policy, London, The CAB International.

${ }^{7} \mathrm{La}$ PAC es una de las áreas más detalladamente reguladas en los Tratados europeos. Véanse al respecto lo artículos 38 al 44 del Tratado de Funcionamiento de la Unión.

${ }^{8}$ Atendiendo a los créditos comprometidos, en el año 2013 la PAC supondrá un 39\% del gasto total del presupuesto de la UE. El gasto dirigido a los pagos a los agricultores se financia íntegramente con cargo al presupuesto comunitario, es el conocido como Pilar I. Las iniciativas de desarrollo rural -el Pilar II-, parte más pequeña que la anterior, son cofinanciadas por los Estados miembros.
} 
La formulación de la PAC y sus distintas reformas se han mostrado como una de las áreas políticas de mayor complejidad en la Unión Europea, donde se produce la colusión de intereses muy diversos: nacionales, económicos e institucionales ${ }^{9}$. Es, incluso, es una de las áreas más fértiles para la acción de los lobbies, representantes de los intereses de los agricultores y de la industria agroalimentaria, que tratan de hacer llegar sus demandas a las instituciones comunes. Dado que el sostenimiento financiero del modelo es exclusivamente comunitario es, además, un área política que entra en conflicto con otras discusiones, especialmente aquella relativa a las Perspectivas Financieras, donde se discuten los presupuestos. Entran así en conflicto las posiciones de Estados con un fuerte sector agrícola que defenderán presupuestos amplios para la UE, como es el caso de Francia, con los que no obtienen tanto beneficio de las asignaciones de la PAC, que pugnan por recortes del presupuesto y de la $\mathrm{PAC}^{10}$. Igualmente los Estados tienden a apoyar aquellas reformas que responden no a necesidades objetivas del conjunto sino a las de sus agriculturas e industrias concretas y, lógicamente los Estados grandes, como Francia en este caso, tienen un mayor peso $^{11}$. Todos estos condicionantes, por tanto, afectan a la definición del modelo de seguridad alimentaria europea en cada momento que no depende exclusivamente de un análisis técnico y político centrado en las necesidades alimentarias europeas.

La PAC, como cualquier atribución política a la UE, se encuentra regulada por el Tratado de Funcionamiento de la Unión (TFUE). Como consecuencia de la reforma del Tratado de Lisboa, la agricultura en su artículo 4 es categorizada como una competencia compartida entre la UE y sus Estados miembros, cuyo ejercicio queda afectado por los principios de proporcionalidad y subsidiariedad. El hecho de que sea una política común no es contradictorio con la competencia complementaria. El amplio ejercicio de la competencia por parte de la UE, en la práctica, ha limitado el papel de los Estados miembros a la ejecución de escasa normativa y a la mera aplicación de lo dispuesto en Bruselas ${ }^{12}$.

Los objetivos que debe cumplir taxativamente la PAC se encuentran recogidos en el artículo 39 del TFUE, que, a efectos prácticos, constituyen el marco político y jurídico para el desarrollo de la competencia $\mathrm{y}$, por tanto, para la definición del modelo de seguridad alimentaria europeo. Estos son: incrementar la productividad agrícola fomentando el progreso técnico, asegurando el desarrollo racional de la producción y el empleo óptimo de los factores; garantizar un nivel de vida equitativo a la población agrícola; estabilizar los mercados; garantizar la seguridad de los abastecimientos; y asegurar al consumidor precios razonables. Además, esta política, como el resto de las comunitarias se ve afectada por una serie de objetivos transversales que deben respetarse al mismo nivel que los específicos: el respeto y protección del medio ambiente (art. 11), la garantía de la salud y seguridad de los consumidores (art. 12), el bienestar de los animales (art. 13) y otras cláusulas sociales como el

\footnotetext{
${ }^{9}$ En relación a esta cuestión puede verse: Yamaç, Necati y Acar, Mustafa: "An análisis of the Obstacles and difficulties in the Introduction of the EU Common Agricultural Policy Reform", METU Studies in Development, no 36 (2009), pp. 423-438.

${ }^{10}$ De hecho, el 14 de septiembre de 2010, el Ministro francés de Agricultura, Bruno Lemaire, y la Ministra alemana de Agricultura, Ilse Aignier, alcanzaron un acuerdo común para defender un PAC potente en el escenario posterior a 2013. Véase al respecto: Europe documents, nº. 2539, (16 Septiembre 2010), pp. 1-4.

${ }^{11}$ La defensa cerrada y constante de los Gobiernos franceses de su sector agrícola no responde sólo a la presión de los grupos de interés, sino sobre todo la defensa de Francia como gran nación que en defensa de esa grandeza debe tener una presencia significativa en los mercados agrícolas internacionales. Véase: Vleuten, Anna van der y Alons, Gerry: "La Grande Nation and Agriculture: The Power of French Farmers Demystified", West European Politics, vol. 35, no 2 (2012), pp. 206-285.

12 Sobre la naturaleza de las competencias compartidas, véase: Hinojosa Martínez, Luis Miguel (2006): El reparto de competencias entre la Unión Europea y sus Estados miembros, Valencia, Tirant lo Blanch, pp. 91-97.
} 
pleno empleo, protección social y formación cualificada o la igualdad entre mujer y hombre (arts. 7 y 8 ).

En la definición y aplicación del modelo de seguridad alimentaria europeo en la actualidad el protagonismo lo tienen las tres instituciones comunitarias que conforman el conocido triángulo en la decisión comunitaria. La Comisión tiene la iniciativa legislativa con carácter exclusivo y la normativa será fruto de la aprobación tanto del Consejo por mayoría cualificado, como del Parlamento. Hoy ha quedado desplazado el tradicional peso de los Estados miembros, ya que antes el Parlamento no intervenía y la mayoría de las decisiones se alcanzaban por consenso, en aplicación del tradicional Compromiso de Luxemburgo ${ }^{13}$. El fuerte protagonismo de las instituciones comunes y los procedimientos institucionalizados europeos hace que la política se caracterice por ser mayoritariamente estable, cuyas reformas presentan resultados parciales, limitados e incrementales ${ }^{14}$.

\section{Evolución de la Política Agrícola Común: distintos modelos}

La PAC es una de las políticas comunes que antes se pone en marcha, en 1962, dada la necesidad de incentivar la producción de alimentos en Europa y de reducir su dependencia alimentaria del exterior y de dar satisfacción a un potente sector agrícola en Francia. En sus cincuenta años de existencia, la PAC ha evolucionado desde un modelo productivista en los inicios a otro multifuncional en la actualidad de manera suave y no brusca, de forma que todavía quedan algunas de las características de los orígenes. Describamos brevemente esa evolución, donde además estableceremos las características generales que se proponen desde la Comisión para la reforma de 2013 y que conformarán la futura PAC al menos hasta $2020^{15}$.

\subsection{2-1992. El modelo productivista o Modelo Mansholt}

La PAC se pone en marcha tras los acuerdos de la conocida como Conferencia de Stressa, que seguirá el diseño del Comisario neerlandés Sicco Mansholt. En un contexto de Guerra Fría y gran vulnerabilidad alimentaria europea, en que todavía pervive el racionamiento para algunos productos, se opta por un modelo que incentive la producción de alimentos con el fin de posibilitar la disponibilidad de alimentos a precios asequibles. Se decide promover la productividad máxima a través de la intervención pública, que garantiza unos precios mínimos a los agricultores. Así, si los precios del mercado caen por debajo del mínimo garantizado, el sector público retira esa cantidad de producto pagando al agricultor el precio

\footnotetext{
${ }^{13}$ Fruto de una crisis institucional en 1965 relacionada directamente con la PAC, la de la "silla vacía", el Compromiso de Luxemburgo condujo a que, en áreas en que el Consejo decidiera por mayoría cualificada, en la práctica se impusiera el consenso, ya que cuando un Estado argumentara un interés nacional se asumía el compromiso de seguir negociando hasta alcanzar el acuerdo. Véase: Truyol y Serra, Antonio (1999): La Integración Europea: análisis histórico-institucional con textos y documentos. Vol. I: Génesis y desarrollo de la Comunidad Europea (1951-1979), Madrid, Tecnos, pp. 313-316.

${ }^{14}$ Las reformas sostenidas y no bruscas se acomodan bastante al modelo de análisis teórico del institucionalismo histórico. Véase al respecto: Lăçan, Nicoleta: "Can Historical Institutionalism Explain the Reforms of the Common Agricultural Policy?”, Romanian Journal of European Affairs, vol. 12, n 1, (2012), pp. 76-85.

${ }^{15}$ En relación a los distintos modelos y objetivos a los cuales ha respondido la PAC a lo largo del tiempo, véase: Cunha, Arlindo y Swinbank, Allan (2011): An Insider View of the CAP Reform Process: Explaining the MacSharry, Agenda 2000 and Fischler Reforms, Oxford, Oxford University Press; Garzon, Isabelle (2006): Reforming the Common Agricultural Policy: History of a Paradigm Change, Basingstoke, Palgrave Macmillan; Loyat, Jacques (2012): Une PAC pour quels modèles de développement?: histoire et perspectives, Paris, France Agricole.
} 
mínimo establecido. La fijación de precios y subvenciones, la decisión y gestión de la producción y comercialización se pusieron en manos de las llamadas Organizaciones Comunes de Mercado (OCM), organismos mixtos creados para determinados grupos de productos, formados por autoridades públicas, productores, distribuidores y consumidores, con poder decisor y gestor ${ }^{16}$.

Frente al exterior se establece un modelo proteccionista apoyado en el principio de preferencia comunitaria, que sitúa al producto comunitario en mejores condiciones de competitividad frente al exterior. La preferencia comunitaria también dependía de una fuerte intervención pública, ya que por los precios mínimos garantizados en el interior y el querer ofrecer al agricultor una renta suficiente los precios de producción en el seno de la entonces CEE eran mucho más elevados que las de otros países, especialmente los países menos desarrollados. Era, por tanto, preciso establecer mecanismos para equiparar los precios de los mercados internacionales a los europeos, y viceversa. El primero de ellos son los derechos de exacción variable -prélèvements- para los productos procedentes de la importación ${ }^{17} \mathrm{y}$, el segundo, la subvención a los exportadores para poder colocar los productos agrícolas europeos en los mercados exteriores a precios competitivos.

Este modelo productivista alcanzó pronto sus objetivos, consiguiendo no sólo la autosuficiencia europea sino incluso situar a la CEE como un importante exportador de productos agrícolas. Sin embargo, también produjo importantes consecuencias negativas. Incentivó que, en aras de la productividad, la mayor parte de la agricultura y ganadería europeas fueran de tipo intensivo, con importantes efectos medioambientales como la degradación de suelos, la contaminación de tierras y aguas por el uso descontrolado de fertilizantes e insecticidas y la sobreexplotación de acuíferos en determinadas zonas. También llevó a la sobreproducción y al exceso de stocks públicos de alimentos que no se sabía como colocar.

En consecuencia también supuso un importante coste financiero para la Comunidad, que a inicios de los 80 llegaba a destinar hasta el $70 \%$ del conjunto del presupuesto comunitario a sufragar la PAC. Esta política también era objeto de importantes críticas en el contexto internacional, que presionaba por una liberalización de los mercados internacionales, especialmente por parte de los países en vías de desarrollo, cuya principal producción para la exportación es de tipo agrícola. Los efectos negativos llevarían a que en los años 80 se adoptaran algunas pequeñas correcciones pero sin alterar el modelo. Se trataba fundamentalmente del conocido como sistema de cuotas que, para determinados productos sobreproducidos, limitaba a una cantidad establecida la garantía de los precios tratando de limitar la oferta. Serían así famosas en las negociaciones agrícolas europeas las discusiones sobre la cuota láctea, vacuna, la del azúcar o los viñedos.

\footnotetext{
${ }^{16}$ Las OCM y sus funciones aparecen reguladas en el artículo 40 del TFUE. Se crearon hasta 21OCMs dividas según los productos o grupos de productos, como por ejemplo, frutas y verduras, cereales, vino, aceite..., llegando a cubrir el $90 \%$ de la producción europea. A partir de 2007, cuando ya se está consolidando el desacoplamiento de la subvención sobre la producción, yendo a un sistema de pago único, se decidió simplificar la gestión de la PAC sustituyendo las 21 OCMs existentes por una única OCM, encargada de la gestión del conjunto de la PAC. Las distintas OCMs son responsables de la regulación concreta de cada uno de los sectores tanto en relación a la producción como a la comercialización. Véase al respecto: Mögele, Rudolf y Erlbacher, Friedrich (eds.) (2011): Single Common Market Organisation, Munich, Verlag C.H. Beck.

${ }^{17}$ Se trata de aranceles variables porque buscaban equiparar el precio internacional del producto importado al precio que hubiera alcanzado ese mismo producto en el mercado comunitario. Dependían, por tanto, en cada momento de los diferentes niveles de precios y no eran fijados a través de un porcentaje como suele ser lo normal en el caso de los aranceles.
} 


\subsection{2-2002. Del producto al productor: la reforma de McSharry}

En 1992 el entonces Comisario de Agricultura, el irlandés Ray MacSharry, perteneciente a la segunda Comisión Delors, puso en marcha la tan necesaria reforma de la PAC para tratar de corregir los efectos perversos derivados del modelo puramente productivista e intentar adecuar la PAC a los debates internacionales sobre comercio de productos agrícolas. El objetivo fundamental era desincentivar la producción a través de dejar de sostener los precios de los productos en sí, para pasar a subvencionar directamente a los productores en su función de su capacidad para garantizar la viabilidad de la agricultura. Se pasa de garantizar precios a dar subvenciones a un tanto alzado sobre las unidades de producción de cada explotación agrícola o ganadera. Se buscaba así un doble beneficio: por una parte, estabilizar y progresivamente reducir el gasto presupuestario destinado a la PAC y, por otra, garantizar la renta agrícola, como dispone el Tratado.

Desde es el punto de vista internacional se trataba también de alinear la PAC con los resultados que se anticipaban de las discusiones internacionales y luego se lograron en el Acuerdo sobre Agricultura del GATT, incluido dentro de los Acuerdos de Marrakech de 1994. Era, por tanto, necesario reducir la intervención pública vía subvenciones y facilitar el acceso de productos agrícolas procedentes de terceros Estados a los mercados comunitarios para entrar dentro de los estándares internacionales.

Esta reforma integra la preocupación medioambiental iniciando el proceso conocido como greening, con la finalidad de promover formas extensivas de agricultura y un modelo sostenible. Así, la normativa, por ejemplo, exigía a las explotaciones agrícolas, según su tamaño, que dejen un determinado tiempo un porcentaje de sus tierras en barbecho para permitir la recuperación del suelo. Se incentivan mediante subvenciones el destino de tierras a pastos, la reforestación o la diversificación de cultivos. De esta época data también la primera legislación sobre agricultura ecológica, su definición, requisitos y la concesión de una etiqueta de calidad.

A partir de mediados de la década de los 90 otro de los objetivos transversales entra con fuerza en la PAC, la preocupación por la seguridad de los alimentos y sus repercusiones sobre la salud de los consumidores ${ }^{18}$. Consecuencia de la crisis conocida como de las "vacas locas", la Comunidad Europea iniciará un potente desarrollo normativo de las condiciones de producción de alimentos agrícolas y de piensos para ganadería destinada a consumo humano. Se pone en marcha así el proceso conocido como "de la granja a la mesa", que va a garantizar a través de la trazabilidad y controles administrativos exhaustivos de la red europea de seguridad alimentaria, el cumplimiento de los estándares normativos en toda la cadena de producción y distribución alimentaria. En este ámbito la UE cuenta en este momento con los estándares de protección más altos del mundo, que se exportan reflexivamente ya que deben demandarse mediante controles en frontera a los productos procedentes de terceros Estados.

La reforma MacSharry también iniciará dos vías nuevas: el fomento de la competitividad de la agricultura europea y la conocida como orientación al mercado. La competitividad del sector agrícola se fomenta mediante la incentivación de la formación de los agricultores y medidas de apoyo a la incorporación de jóvenes a la actividad. El objetivo de la orientación al mercado puede encontrarse en la promoción de la llamada agricultura de calidad a través de la adopción de normativa que acuña las etiquetas de Denominación de Origen, garantizadas a través de Consejos Reguladores que aseguran que todos los alimentos

\footnotetext{
${ }^{18}$ Ya se ha ofrecido bibliografía sobre la política de seguridad alimentaria de la UE, véase supra nota 2.
} 
respaldados por esa etiqueta responden a las exigencias de calidad superior de esa Denominación. En la misma senda, la Agenda 2000 que profundiza la nueva orientación MacSharry promovería el desarrollo rural a partir de la diversificación de actividades en el campo, incrementando la financiación destinada al llamado Pilar II de la PAC ${ }^{19}$. Se busca así que el campo no sólo viva de la agricultura sino que se desarrollen actividades complementarias como el turismo rural, industrias agroalimentarias, artesanías, servicios culturales, etc.

Con esta reforma se inicia el camino hacia la multifuncionalidad de la PAC, que integra principios diferentes como el medioambiental, el de producción de alimentos sanos, seguros y saludables, la producción ecológica y de calidad o la diversificación de actividades. Sin embargo, en esta época la dimensión productivista todavía está muy presente, a través del pago por hectárea o cabeza de ganado como elemento fundamental de la PAC.

\subsection{3-2013. La multifuncionalidad. La reforma Fischler}

En 2003 el Comisario de Agricultura austríaco Franz Fischler propondría una gran reforma de la PAC, que es el modelo actualmente en vigor $^{20}$. La PAC responde en el siglo XXI a un modelo multifuncional ya que busca sostener al agricultor por su función como agente social $^{21}$. No se considera al agricultor sólo como productor de alimentos sino como proveedor de "bienes públicos globales", siendo estos de tipo medioambiental, social, económico, cultural, etc. Se persigue que la producción de alimentos se haga con una buena gestión de los recursos, se garantice la viabilidad de las áreas rurales y se considera también positiva la contribución de la agricultura a la lucha contra el cambio climático, en la medida en que las explotaciones actúan de sumideros de $\mathrm{CO}_{2}$.

Debe sostenerse la actividad agrícola porque el abandono del campo tendría consecuencias nocivas para el medioambiente como la desertificación, además de sociales como fuertes presiones derivadas de la emigración masiva campo-ciudad. Desde un punto de vista económico, la agricultura competitiva no sólo crea empleo en el ámbito rural, sino que además genera otras industrias y contribuye a la vertebración territorial. Finalmente, también es preciso tener el cuenta la importancia del agricultor y la población rural en la conservación de las culturas tradicionales de los Estados miembros, tradiciones orales, forma de vida y costumbres, manifestaciones artísticas, artesanías, gastronomías locales, etc. Son todos estos bienes públicos los que la PAC debe ahora sostener y promover, siendo una política para el desarrollo social.

La PAC sigue apoyada en las subvenciones pero estas se calculan de manera diferente, en un proceso prolongado conocido como "desacoplamiento". La subvención a los productores se entiende como un complemento a las rentas que obtienen de vender sus productos en unos mercados cada vez más libres y menos dependientes de la intervención de precios. Ese apoyo de la PAC ahora es un pago directo calculado en función a unos datos objetivos determinados en base a una referencia histórica. Este desacoplamiento buscaba

\footnotetext{
${ }^{19}$ Véase: Serger, Sylvia Schwaag: "Negotiating CAP Reform in the European Union - Agenda 2000

" Swedish Institute for Food and Agricultural Economics, Report 4 (2001).

${ }^{20}$ Para una valoración de esta reforma y sus efectos: Sorrentino, Alessandro, Henke, Roberto y Severini, Simone, (eds.), (2011): The Common Agricultural Policy After the Fischler Reform: National Implementations, Impact Assessment and the Agenda for Future Reforms, Farnham, Ashgate.

${ }^{21}$ Una buena descripción sobre qué se entiende por carácter multifunctional de la agricultura europea, puede encontrarse en el siguiente documento de reflexión de la Comisión Europea: "Why do we need a Common Agricultural Policy?”, European Commission, Directorate-General for Agriculture and Rural Development, Discussion Paper by DG Agriculture and Rural Development, Brussels (Diciembre 2009).
} 
principalmente adecuar la PAC a los requerimientos del marco comercial internacional de la $\mathrm{OMC}^{22}$.

La PAC pone más énfasis en la dimensión medioambiental a través de la conocida como "ecocondicionalidad", de forma que la percepción del pago directo se condiciona al cumplimiento de determinados normas medioambientales, de forma que la agricultura que no respete determinados usos verdes no pueda beneficiarse de la financiación de la PAC. También se refuerza el apoyo al agricultor para su orientación al mercado, no sólo a través del apoyo a la formación y la adquisición de nuevas competencias, sino a través de medidas que incentivan nuevos productos demandados por el mercado, una industria alimentaria innovadora y se impulsa la agricultura ecológica de calidad, dirigida a consumidores exigentes.

Consecuencia de la aplicación de los acuerdos internacionales, se reducen sustancialmente los subsidios a la exportación lo que también requiere que se apoye al agricultor de otras maneras para conseguir ser competitivo en los mercados internacionales. También se ha continuado con el fomento de alternativas de desarrollo en el entorno rural, reforzando la financiación destinada al Pilar II de la PAC. En el año 2008 se desarrolló el conocido como Health Check, con la finalidad de evaluar los nuevos desafíos a los que se enfrenta la PAC tanto derivados de las presiones internas como internacionales ${ }^{23}$. Se acordó así la necesidad de reforzar el objetivo de la gestión de riesgos para conseguir que el agricultor sea menos dependiente de los azares atmosféricos, de plagas y de fluctuación de los precios de los alimentos. Su diagnóstico debe conducir a la mejora normativa de la próxima reforma.

\section{Los resultados de las reformas. La PAC hoy en día}

Analizando la evolución de la PAC, se ha avanzado hacia un concepto de la misma multifuncional, ya que ésta política busca promover otros objetivos además de la seguridad alimentaria de la población europea y una renta justa para el sector agrícola ${ }^{24}$. Se ha abandonado el modelo productivista de los inicios, pero tampoco se ha sustituido por un modelo estrictamente neoliberal. La PAC sigue siendo un sector altamente regulado e intervenido, donde la producción de alimentos no depende sólo de las leyes de los mercados internacionales. Se interviene para garantizar determinados bienes públicos. Primero la producción de alimentos, que sean altamente seguros y saludables para los consumidores, producción muy respetuosa con el medio ambiente. Además, la PAC también debe proveer otros bienes públicos, económicos, de empleo, sociales y culturales. Ha pasado de afectar al $71 \%$ del total del presupuesto comunitario en 1984 a destinarse un 39\% del presupuesto total previsto para 2013.

\footnotetext{
22 Swinbank, Alan y Daugbjerg, Carsten: "The 2003 CAP Reform: Accommodating WTO Pressures", Comparative European Politics, vol. 4, no 1 (2006), pp. 47-64.

${ }^{23}$ Para un análisis de esta cuestión: Daugbjerg, Carsten y Swinbank, Alan: "Explaining the 'Health Check' of the Common Agricultural Policy: budgetary politics, globalisation and paradigm change revisited", Policy Studies, vol 32, no 2 (2011), pp. 127-141.

${ }^{24}$ Un interesante trabajo sobre los conceptos jurídico-políticos y su conexión con los paradigmas defendidos puede verse en: Petrick, Martin: “The Co-evolution of Semantics and Policy Paradigms: 50 Years of Europe's Common Agricultural Policy, Intereconomics, vol. 43, no 4 (Jul-Ago. 2008), pp. 246-252.
} 
Los resultados de las reformas se ven con claridad en clave evolutiva a partir del análisis de una serie de gráficas. La gráfica 1 nos muestra el efecto de los distintos modelos analizados sobre los precios. Tomando como referencia el precio del trigo y los precios estadounidenses como indicativos de precios internacionales, puede verse como en la década de los ochenta, una PAC volcada en el fomento de la producción a través del sostenimiento de precios, tiene como consecuencia unos precios del trigo europeos estables, y artificialmente elevados, llegando a alcanzar en 1986 más del doble del precio de Estados Unidos. A partir de 1993 puede percibirse ya el efecto de la reforma MacSharry, con un descenso sustancial de los precios europeos, de más del $25 \%$, que los mantienen mucho más cercanos aunque todavía sensiblemente superiores a los de los precios estadounidenses. Igualmente en este periodo se ve que los precios muestran unas mayores oscilaciones, consecuencia de que son más sensibles a las fuerzas de los mercados al desaparecer el sostenimiento artificial. Después de 2003, cuando empieza a implementarse la reforma Fischler, puede verse ya unos precios prácticamente iguales a los estadounidenses y que sufren las mismas oscilaciones, consecuencia de las tensiones de los mercados internacionales.

\section{Figura 1. La evolución del papel del sostenimiento de los precios. Caso del trigo (en precios nominales. Año de mercado, de julio a junio) \\ EUR/ton}

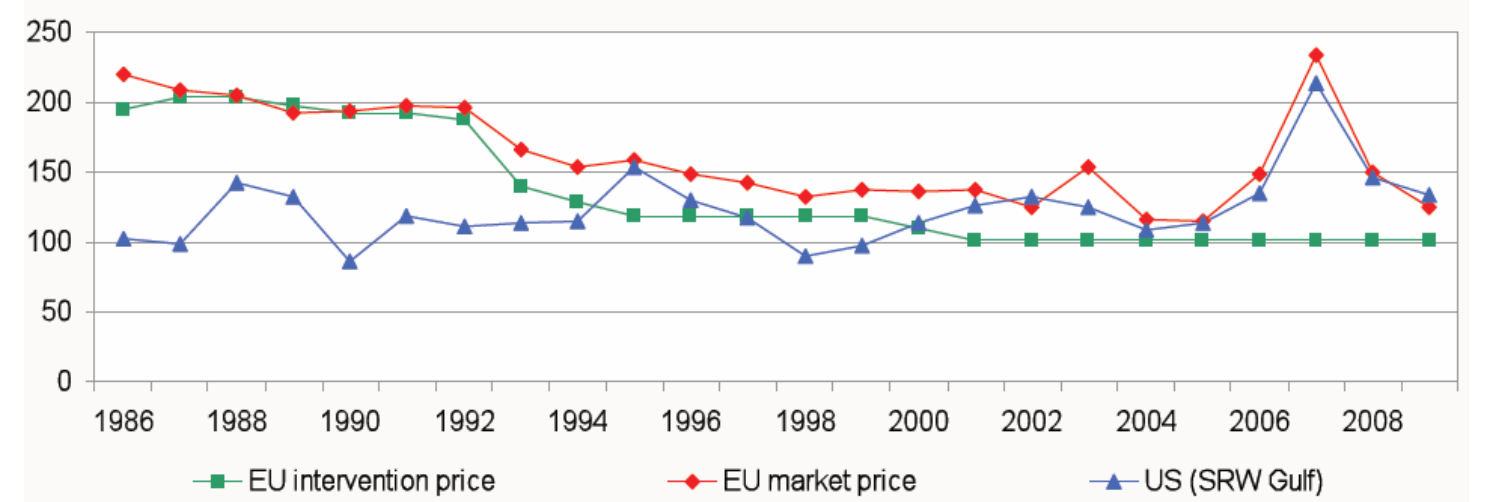

Sources: DG Agriculture and Rural Development and World Bank.

Fuente: DG Agriculture and Rural Development y Banco Mundial

La figura 2 detalla en distintos colores la composición del gasto de la PAC a lo largo del tiempo y su evolución consecuencia de las distintas reformas estudiadas. Puede verse como en 1980 todo el gasto de la PAC estaba dividido en partes iguales entre el sostenimiento de precios (en amarillo) y la subvención a la exportación (en rojo). En 1991 comenzaría tímidamente el apoyo al desarrollo rural (color lila), sobre todo a través de medidas de modernización de las explotaciones. Como consecuencia de los compromisos internacionales suscritos, las subvenciones a la exportación comienzan con una tendencia a la reducción hasta su casi desaparición en 2008. Si miramos la garantía de la PAC, puede verse como desde 1992 el sostenimiento directo de precios a través del mecanismo de los precios mínimos garantizados (en amarillo), se ve progresivamente desplazada por los pagos directos (en azul). En 2006 con la entrada en vigor de la reforma Fischler, los pagos directos a su vez son progresivamente reemplazados por los pagos desacoplados (en verde), que ya no dependen de la capacidad de producción. El escaso $8 \%$ de sostenimiento de precios que queda en 2009 se debe a la pervivencia de cuotas para productos concretos (leche, azúcar y viñedos) destinados a extinguirse en 2013. 


\section{Figura 2. La evolución de la PAC (en \%)}

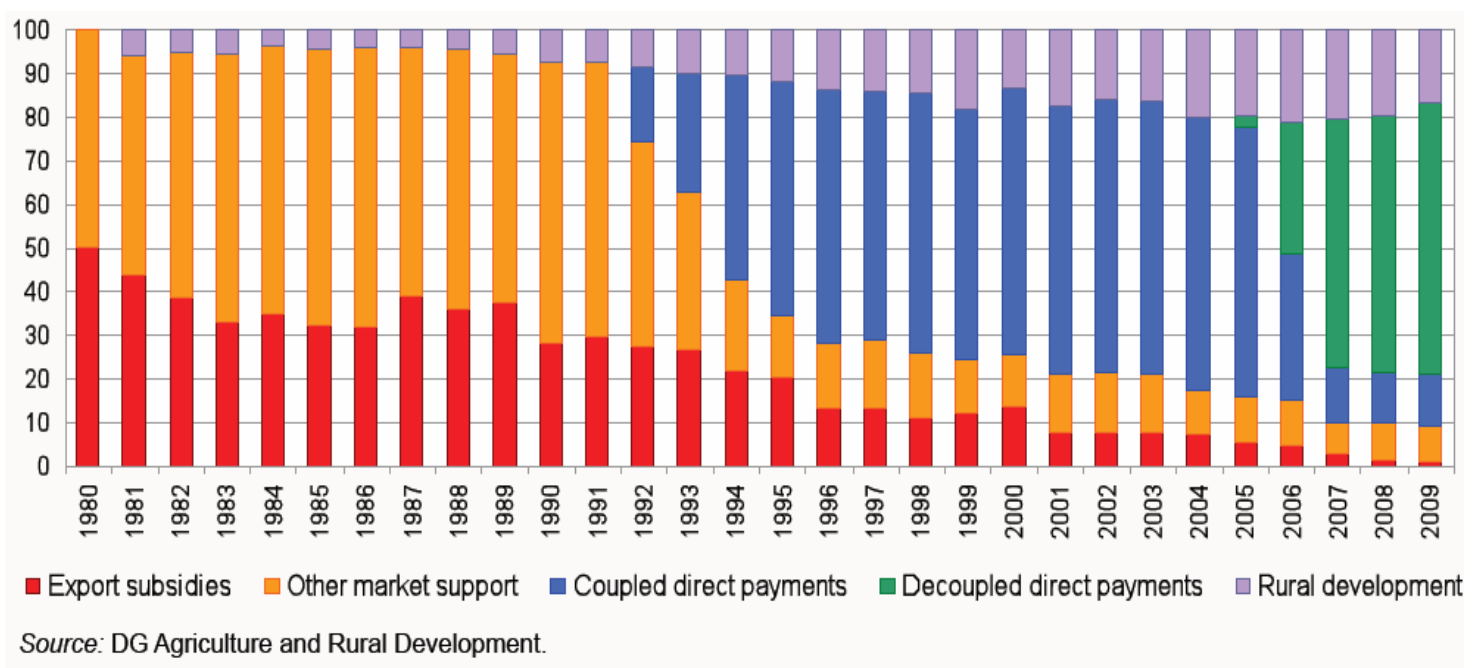

Fuente: DG Agriculture and Rural Development y Banco Mundial

En tercer lugar, si uno de los problemas clásicos del modelo productivista de la PAC era la superproducción y los excedentes de productos, que acarreaban un sobrecoste de almacenamiento y, a veces incluso, de destrucción, podemos preguntarnos por los efectos en este capítulo. Puede verse cómo los excedentes medios del periodo 1990-1994 se han reducido notablemente respecto a los del periodo 2005-2009 en todos los casos estudiados por efecto de las reformas. Es reseñable el caso del vacuno, en que se ha pasado de ser excedentario en más de un $10 \%$ a depender de las exportaciones en un $4 \%$.

Figura 3. Impacto de las reformas PAC en el superávit neto de producción

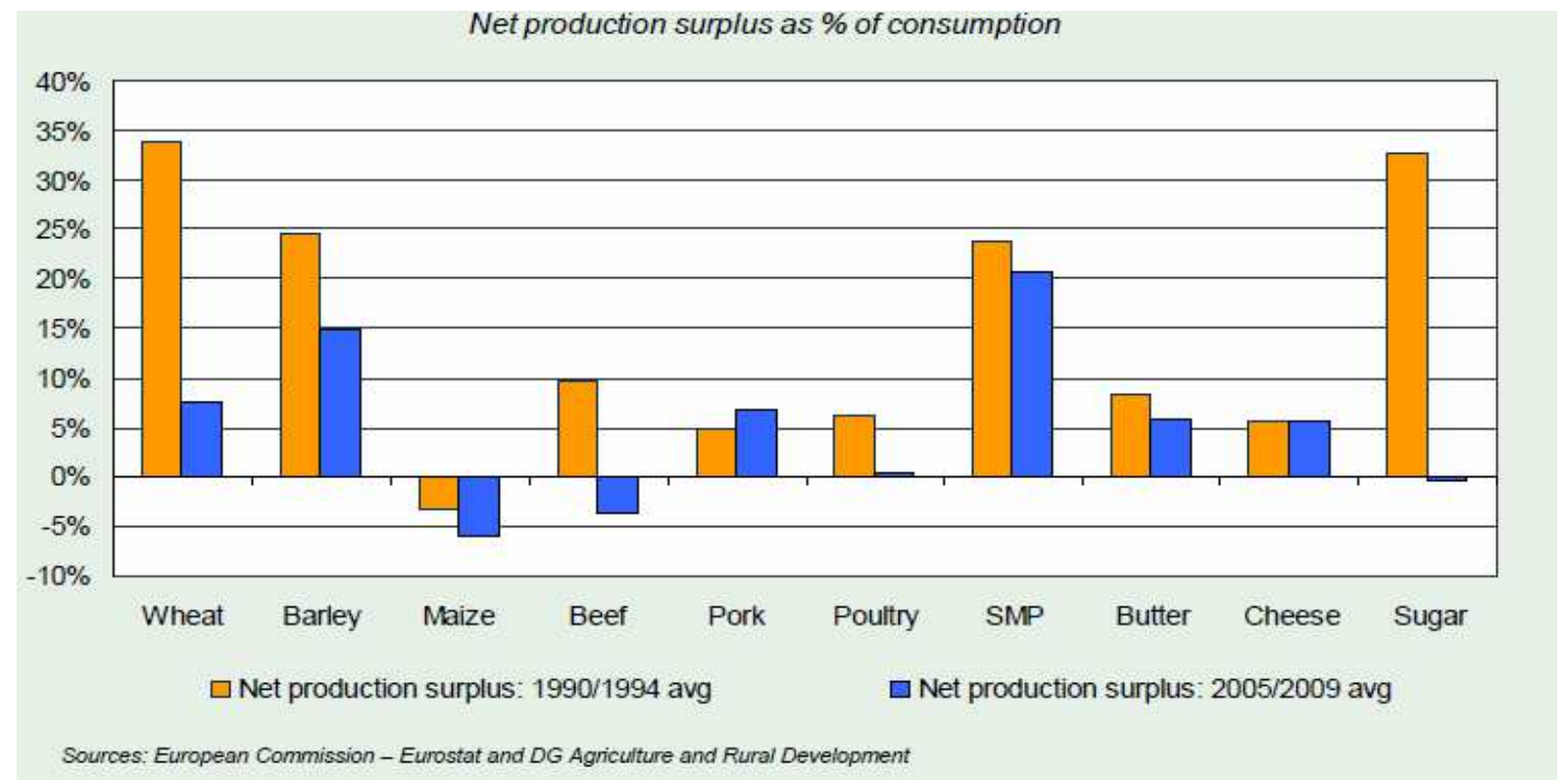


Fuentes: Comisión Europea y DG Agriculture and Rural Development

A modo de resumen, el cambio de modelo de la PAC, de uno productivista a otro multifuncional ha supuesto, en primer lugar, una reducción muy sustancial de la carga que supone la PAC para el contribuyente europeo. Se ha producido una reducción de precios muy notable cercana al 50\%, como consecuencia de la "no intervención", que los aproximan a los precios de los mercados internacionales. Si han caído los precios de los alimentos y los consumidores no hemos notado esa disminución en nuestros bolsillos, cabe deducir que se ha multiplicado el coste de la distribución y la transformación en la cadena alimentaria. También hay que subrayar la disminución de los superávits de exportación de los productos sostenidos, consecuencia de la no subvención y la reducción de los stocks de alimentos intervenidos.

Finalmente, la práctica eliminación de las medidas que distorsionan el comercio de alimentos, permiten el cumplimiento de compromisos internacionales y la entrada de alimentos de terceros países en los mercados comunitarios. Esto permite reforzar la confianza de nuestros socios comerciales. Sin embargo, también hay que mencionar otro efecto, no tan positivo y en buena medida inesperado. La incorporación a partir de la segunda mitad de los 2000 de una cantidad reseñable de producto UE a los mercados internacionales sin subvención, y por tanto sin bajar el precio del producto europeo, ha tenido como consecuencia un incremento de los precios internacionales de los alimentos, al haber entrado una gran cantidad de producto a precio alto en los mercados.

\section{Las propuestas de reforma de la PAC 2014-2020: :.Qué modelo para un futuro inmediato?}

No podemos finalizar la parte relativa al análisis del modelo de seguridad alimentaria de la UE, sin advertir que está encima de la mesa una nueva reforma de la misma, aún en proyecto y todavía no aprobada. Las razones para la reforma son muchas y variadas y, en buena medida, condicionarán el sentido y contenido de las nuevas medidas políticas ${ }^{25}$.

El primer factor para la revisión viene del hecho de que a partir de 2014 entramos en un nuevo marco financiero europeo, el que recojan las Perspectivas Financieras 2014-2020, que son las primeras que afectan desde sus inicios a una Unión de 27 Estados miembros. Las nuevas Perspectivas exigen una nueva programación de la política ya que, al financiarse la PAC íntegramente con cargo al presupuesto comunitario, determinarán nuevas cantidades a asignar y hay que decidir qué financiar y, por tanto, las prioridades políticas y el modelo de la PAC $^{26}$. Además, los nuevos Estados miembros, que se adhirieron en 2004 y 2007 piden más equidad, ya que su sector agrícola es tratado con diferencias y percibe cantidades menores que los agricultores de los 15 viejos Estados. Un reparto más equitativo exigirá concentrarse en las prioridades, ya que no podemos obviar que con la ampliación se ha incrementado

\footnotetext{
${ }^{25}$ Los factores y vectores de la reforma fueron planteados por la Comisión en la siguiente Comunicación: Comisión Europea: "Comunicación de la Comisión al Parlamento Europeo, al Consejo, al Comité Económico y Social y al Comité de las Regiones "La PAC en el horizonte de 2020: Responder a los retos futuros en el ámbito territorial, de los recursos naturales y alimentario", Bruselas, COM (2010) 0672/2). El Parlamento Europeo valoró cada uno de los extremos en una Resolución de junio de 2011. Véase: Parlamento Europeo: "Resolución sobre la PAC en el horizonte de 2020", (23 de junio 2010), (P7_TA(2011) 0297).

${ }^{26}$ Sobre los factores y los elementos que necesariamente debe abordar la reforma, véase: Buckwell, Allan: "A CAP Fit for the 21st Century", Rivista di Economia Agraria, vol. 63, no 3 (2008), pp. 313-340; Bureau, JeanChristophe y Mahé, Louis-Pascal: "CAP Reform beyond 2013: An Idea for a Longer View", Notre Europe, Studies and Research, $\mathrm{n}^{\circ} 64$ (2008).
} 
notablemente la población agrícola y tendremos que repartir menos dinero entre más beneficiarios.

La segunda razón para abordar la reforma viene de la necesidad política de legitimar socialmente la PAC. La crisis económica europea ha multiplicado el eco del discurso político contra el hecho de que se destine un $40 \%$ del presupuesto comunitario a financiar una política que sólo beneficia a como mucho un $10 \%$ de la población, la agrícola. Se ha repetido una y otra vez que, en cambio, el presupuesto comunitario debe destinarse a financiar políticas que refuercen la competitividad internacional de la economía europea. Además, existe también una fuerte crítica sobre los aspectos que se financian, habiéndose consolidado la idea entre la ciudadanía que se subvenciona la siembra de unos campos que luego el agricultor ni siquiera se preocupa en recoger.

El tercer elemento que resulta necesario abordar a través de esa reforma es la volatilidad de los precios en los mercados internacionales, volatilidad que entra con fuerza con la crisis de 2008 pero que es una tendencia que tiene visos de perpetuarse. Este elemento reintroduce en el discurso política la preocupación por la seguridad alimentaria, entendida en el sentido clásico de velar por la autosuficiencia de un sector estratégico. También pone encima de la mesa la vulnerabilidad de los agricultores, que sufren directamente las caídas de los precios, y cuya renta habrá que apoyar con esfuerzos más costosos.

Tanto la reforma de la PAC como la volatilidad de precios han puesto al descubierto un problema estructural del sector alimentario europeo como son las desigualdades en la cadena alimentaria. Los agricultores reciben baja remuneración por su producción pero los consumidores pagan precios elevados. La subida de precios en 2008 repercutió rápidamente en los bolsillos de los consumidores, pero cuando se produce la bajada los precios de los alimentos se mantienen constantes para el consumidor último. Resulta, por tanto, fundamental controlar el margen de actuación de distribuidores e industrias alimentarias, a fin de garantizar que la PAC cumple con su objetivo de garantizar precios razonables al consumidor.

Finalmente, el cuarto vector busca revisar la PAC con la vista puesta en el logro de los otros objetivos de la UE, "bienes públicos", y por tanto responder mejor a esa multifuncionalidad. Entre ellos puede citarse la contribución de la PAC a la competitividad económica de la UE y al empleo, aplicando la Estrategia 2020; la lucha contra el cambio climático y la garantía del equilibrio territorial entre todas las regiones europeas y la cohesión social.

Para dar respuesta a todas estas necesidades objetivas la Comisión presentó en octubre de 2011 una propuesta de reforma que en la actualidad todavía está siendo debatida ${ }^{27}$. Se trata de un complejísimo paquete de medidas que aquí no vamos a detallar y analizar una por una, sino que nos limitaremos a apuntar la dirección de las reformas propuestas y su incidencia en el modelo de seguridad alimentaria, teniendo en cuenta que su carácter no es definitivo.

Podemos analizar las medidas propuestas distinguiendo la finalidad de las mismas. En relación a los pagos directos que reciben los agricultores, se pretende conseguir la

\footnotetext{
${ }^{27}$ Para un análisis detallado de esas propuestas y sus consecuencias para el modelo de la PAC, véase: Mahé, Louis-Pascal: "Le projet de la PAC pour l'après 2013 annonce-t-il une "grande" reforme?", Notre Europe Policy Paper (Marzo 2012); D’Oultremont, Clémentine: “The CAP post-2013: more equitable, green and marketoriented?", EGMONT European Policy Brief, no 5 (2011).
} 
convergencia entre los Estados nuevos y los viejos ${ }^{28}$. Además se propone un nuevo pago básico para todos los productores que tendrá un nuevo componente "verde", exigiendo que destinen parte de su superficie de cultivo a usos ecológicos. Se busca una mayor subjetivación de los beneficiarios, premiando el acceso a la profesión por parte de agricultores jóvenes. Se prevén también nuevas reglas para los pagos acoplados y una modificación de la ecocondicionalidad, que se hace más dura. Sobre las medidas de mercado, se confirma el fin de las cuotas. Además, y esto es muy interesante, unos fondos provisionales que se crearon para responder a la crisis de 2008 se extienden a todos los productos como una especie de fondos anticrisis que entrarán en funcionamiento cuando un sector sufra un shock abrupto. Se prevén medidas legislativas para mejorar el funcionamiento de la cadena alimentaria y otra legislación para apoyar la producción de calidad.

Finalmente, en lo que atañe al desarrollo rural se establecen nuevas prioridades y también la convergencia entre Estados miembros. También se quiere que haya una mejor coordinación entre este fondo de desarrollo agrícola y otros fondos estructurales como el regional o el que apoya la formación de los trabajadores. Se busca lograr una simplificación de las medidas apoyadas y a través de este eje, reforzar las herramientas de gestión de riesgos derivadas de factores atmosféricos o económicos al alcance de los consumidores. Además, se proponen la puesta en marcha de Partenariados para la Innovación, con la finalidad de incentivar el carácter innovador y puntero económicamente de la agricultura europea.

En el conjunto de las Perspectivas Financieras, la propuesta de la Comisión no supondría una reducción sustancial del peso de la PAC, sino que mantendría su importe nominal (435.600 millones de euros). Creemos que la decisión final posiblemente se vea alejada de este objetivo, dados los resultados de la Cumbre especial de 22 y 23 de noviembre de 2011, donde parece que se imponen a futuro las tesis de los Estados que quieren un presupuesto comunitario menor, con un peso más pequeño de la $\mathrm{PAC}^{29}$.

Valorando las propuestas de la Comisión, vemos que no hay un cambio de modelo sino que se continúa con la filosofía Fischler, buscando una mejor distribución de recursos, reforzar la orientación al mercado del sector agrícola europeo y el greening, ya que llegan a destinarse hasta el 30\% de los pagos directos a la promoción de objetivos medioambientales. Subrayaríamos algunos de los más significativos elementos innovadores como es la creación de Fondos para responder a emergencias en un determinado sector, o fondos anticrisis, que creemos que verán reforzada su importancia dada la previsible creciente volatilidad de $\operatorname{precios}^{30}$. Igualmente parece positivo que se confirme la finalización de las cuotas y el sostenimiento de precios para la leche, el azúcar y los viñedos.

\footnotetext{
${ }^{28}$ Estas medidas se proponen en el siguiente documento de la Comisión: "Proposal for a Regulation of the European Parliament and of the Council establishing rules for direct payments to farmers under support schemes within the framework of the common agricultural policy", European Commission Brussels, (COM(2011) 625/3). Un análisis detallado de las propuestas puede encontrarse en: Swinbank, Alan: "New Direct Payments Scheme: Targeting and Redistribution in the Future CAP”, European Parliament Studies, PE 474.528 (2012).

${ }^{29}$ La Cumbre especial sobre las Perspectivas Financieras finalizó sin acuerdo el 23 de noviembre de 2012. Los Estados miembros aprobaron una declaración conjunta en la que mandataban al Presidente del Consejo Europeo y al de la Comisión Europea a que siguieran realizando consultas con los Estados miembros a fin de alcanzar un acuerdo a comienzos de 2013. Una de las fracturas que impidieron el consenso fue la originada por el apoyo de Francia a las tesis de la Comisión en materia de la PAC y el rechazo del Reino Unido a mantener el actual nivel de financiación de la PAC. Véase al respecto: "Declaración de los miembros del Consejo Europeo", Consejo Europeo Bruselas, (23 de noviembre de 2012).

${ }^{30}$ Véase en este mismo número los análisis relativos a la volatilidad de precios, desarrollados por Antonio Marquina y Alberto Priego.
} 
Sigue encima de la mesa la limitación de los pagos a las grandes explotaciones que, si parece coherente con los objetivos sociales de la PAC, encuentra grandes reticencias en el importante sector agroindustrial francés. Puede que muchos echen en falta en esta reforma la reducción de las barreras comerciales que hacen que los precios europeos sean más altos, pero lo que está claro es que esta reforma demandada por los países en vías de desarrollo no se adoptará hasta que no haya un acuerdo global en el seno de la Ronda de Doha de la OMC, ya que la UE debe mantener sus herramientas de negociación.

Tenga el alcance final que tenga la reforma, la lectura política es que continúa y no altera el modelo multifuncional de la reforma Fischer. Profundiza y refuerza los elementos definitorios de esta pero no altera las bases del modelo. En el debate político general se había planteado la posibilidad de una renacionalización de la PAC, las propuestas encima de la mesa no van en ese sentido. Tampoco en el de una liberalización total, sino que existe un consenso suficiente sobre el enfoque multifuncional. Ahora bien, dado el contexto internacional que se analiza y describe en este número, las presiones demográficas, las derivadas del cambio social y el factor cambio climático, podemos preguntarnos si es políticamente oportuno mantener el mismo modelo o hay que apostar por otros modelos.

\section{La disponibilidad mundial de alimentos y la dimensión exterior del modelo de seguridad alimentaria de la UE}

La producción mundial y la disponibilidad de alimentos para la población son enormemente heterogéneas a lo largo del planeta. No sólo dependen de factores económicos y naturales, sino sobre todo es el resultado de políticas, y no sólo las políticas que pueda realizar cada uno de los Estados por sí mismo. En este sentido, la UE es un actor muy relevante para la disponibilidad global de alimentos, dado que es un gran productor y un gran mercado, es el segundo exportador mundial de productos agrícolas -sólo después de Estados Unidos- y el primer importador mundial, muy lejos de sus competidores directos ${ }^{31}$. Su política interna, la PAC, y las modificaciones que esta va introduciendo tienen repercusiones directas para la seguridad alimentaria de otros Estados, especialmente los más vulnerables, los países en vías de desarrollo. De las decisiones de la PAC dependen los alimentos disponibles en los mercados internacionales, su precio y, por tanto, las políticas de seguridad alimentaria de terceros Estados. También depende de esa política sus expectativas de exportar o no al principal mercado internacional, el europeo.

Las repercusiones que la actuación de la UE puede tener para las políticas de seguridad alimentaria de terceros Estados puede examinarse desde tres puntos de vista complementarios y todos necesarios. En primer lugar, puede examinarse el reflejo exterior de la PAC, esto es,

${ }^{31}$ El balance neto en el comercio de alimentos es globalmente positivo para la UE. Como exportador, los productos agrícolas y alimentarios suponen el 7\% de las exportaciones globales de la UE, siendo el quinto grupo de productos en importancia. Se exporta, sobre todo, productos finales como vino, whiskies y sustancias aromatizantes. Los principales mercados de exportación son Estados Unidos, Rusia, Suiza y Japón. En términos de importaciones, los productos alimentarios constituyen el $6 \%$ de las importaciones europeas globales, importando sobre todo café, frutas, verduras y soja. Los principales países de origen de los productos alimentarios son Brasil, Estados Unidos, Argentina y China. Los datos europeos de comercio de alimentos pueden consultarse los siguientes informes de la Comisión Europea:, "Monitoring Agri-trade Policy. Global and EU agriculture exports rebound", European Commission, Directorate General for Agriculture and Rural Development, $\mathrm{n}^{\mathrm{o}}$ 1-11 (Mayo 2011); "Monitoring Agri-trade Policy. Agricultural Trade in 2011: The EU and the World", European Commission, Directorate General for Agriculture and Rural Development (Mayo 2012). 
las consecuencias que el modelo de la PAC y las nuevas normativas PAC tienen para terceros Estados. Segundo, podemos ver el comportamiento europeo en el comercio internacional, la acción normativa de la UE en el seno de los marcos globales cuyas decisiones tienen relevancia para esta cuestión. En tercer lugar, es preciso exponer las políticas exteriores concretas que la UE formula en relación a terceros Estados y que tienen repercusiones en la seguridad alimentaria y que son fundamentalmente, el comercio y la cooperación para el desarrollo. Esta última dimensión apunta directamente a los países en vías de desarrollo y receptores de ayuda para el desarrollo.

\subsection{La dimensión exterior de la PAC}

Las distintas opciones políticas de la PAC tienen una repercusión directa sobre la seguridad alimentaria de terceros países y, cuando se trata de países en vías de desarrollo, incluso, de su posibilidad de exportar. La opción proteccionista de PAC para preservar la preferencia comunitaria en los productos agrícolas viene de largo siendo tildada de incoherente con la postura general europea de liberalizar el comercio internacional y su compromiso con el desarrollo de todos los países y pueblos ${ }^{32}$. Se afirma, no sin razón, que cuando el objetivo liberalizador europeo y la buena voluntad de la cooperación para el desarrollo chocan frontalmente con intereses europeos, como el agrícola, pasan a ocupar un segundo plano ${ }^{33}$.

El hecho de que las reformas de la PAC limiten las políticas proteccionistas para determinados productos, reduciendo aranceles, permite que países exportadores de estos productos tengan un acceso más fácil al mercado comunitario. Igualmente los subsidios a la exportación europeos perjudican a los países en vías de desarrollo que son objeto de competencia desleal en los mercados internacionales. En los últimos años, las instituciones europeas son especialmente sensibles al impacto de la PAC en los países en vías de desarrollo. Se ha decidido así la elaboración periódica de informes sobre coherencia de las políticas de desarrollo e impacto de la PAC en ese objetivo ${ }^{34}$.

No puede hacerse un análisis homogéneo de los resultados que una determinada reforma de la PAC pueda tener para los países en vías de desarrollo, sino que hay que analizar los efectos concretos caso por caso atendiendo a las circunstancias de cada país ${ }^{35}$. Los países en vías de desarrollo son crecientemente heterogéneos en su dependencia alimentaria y en la importancia que el comercio de alimentos tiene para sus economías internas. Los efectos que tenga para ellos una medida de la PAC dependerán de la composición por productos del comercio que mantengan con la UE, si esos productos están protegidos o no por el régimen de la PAC, y si el país en concreto tiene un acceso preferencial al mercado de la UE. Por ello, de las propuestas de reforma de la

\footnotetext{
${ }^{32}$ No es irrelevante recordar que dos de los objetivos de la Política Exterior europea, regulados en el Tratado son "apoyar el desarrollo sostenible en los planos económico, social y medioambiental de los países en desarrollo, con el objetivo fundamental de erradicar la pobreza" y "fomentar la integración de todos los países en la economía mundial, entre otras cosas mediante la supresión progresiva de los obstáculos al comercio internacional". Véase al respecto el artículo 21 del TUE.

${ }^{33}$ Sobre las incoherencias de la política exterior europea, especialmente entre los objetivos de la cooperación para el desarrollo y los intereses de la Política Agrícola Común, véase: Sotillo Lorenzo, José Ángel (2006): Un lugar en el mundo: la política de desarrollo de la Unión Europea, Madrid, La Catarata, esp. p. 191 y ss.

${ }^{34}$ Véase, a título de ejemplo, la valoración del impacto de la reforma propuesta de la PAC para 2014 en los países en vías de desarrollo: "Commission Staff Working Paper. Impact Assessment. Common Agricultural Policy Towards 2020", European Commission, Brussels, (SEC(2011) 1153 final/2 (20 de octubre 2011).

${ }^{35}$ Véase en relación a esta cuestión: Matthews, Alan: "Post-2013 EU Common Agricultural Policy, Trade and Development: A Review of Legislative Proposals", ICTSD Programme on Agricultural Trade and Sustainable Development, Issue Paper, $\mathrm{n}^{\mathrm{o}} 39$ (2011).
} 
PAC para 2014-2020 no se pueden sacar conclusiones apriorísticas sobre si benefician o perjudican a terceros Estados. Quizás la única apreciación se pueda hacer en el caso de aquellos fuertemente dependientes de los productos sometidos a cuotas que finalizarán. Así, en el caso del azúcar, es lógico esperar que la producción se expanda en el seno de la UE y, por tanto, disminuyan las importaciones de Estados terceros.

\subsection{Acción en los marcos multilaterales globales: OMC y G-20}

La Unión Europea es un actor en las instituciones internacionales que actualmente son relevantes para la seguridad alimentaria global, especialmente en sus dimensiones comerciales (OMC) y de actuación frente a las crisis alimentarias (G20). En estos foros la UE se encuentra representada como un agregado, a la vez que todos o algunos de sus Estados miembros. Conviene examinar, por tanto, su relevancia y su actuación para las políticas globales de seguridad alimentaria.

La UE ha sido un actor fundamental en las relaciones comerciales internacionales desde los años $60 \mathrm{y}$, junto a otros Estados desarrollados como Estados Unidos, jugó un papel clave en los Acuerdos de Marrakech de 1994 que trasformaron el régimen GATT en la Organización Mundial de Comercio ${ }^{36}$. En la posición de negociación europea y, consecuentemente, en el alcance de los acuerdos internacionales en materia de liberalización del comercio, la PAC y sus posibilidades de reforma interna han jugado un papel determinante en el pasado ${ }^{37}$. Ya hemos visto como uno de los principales factores para las sucesivas reformas de la PAC, especialmente, la eliminación de los precios garantizados y la reducción de los subsidios a la exportación provenían de los compromisos internacionales europeos.

Precisamente, en el momento actual de impasse en el seno de la Ronda de Doha, abierta en 2001, los obstáculos para el acuerdo provienen también del ámbito agrícola ${ }^{38}$. Los países en vías de desarrollo exigen de los desarrollados mayores concesiones de acceso a sus mercados y eliminación de subvenciones a la exportación. En los últimos años la UE no está entre los grupos de Estados más beligerantes, el último fracaso negociador de 2008 se debió al conflicto entre Estados Unidos e India ${ }^{39}$. Por el contrario, en los últimos años la UE está mostrando un papel activo proponiendo nuevas concesiones y ofertas de negociación, pero que no han tenido resultados a la hora de impulsar las negociaciones ${ }^{40}$. Se ofreció acabar con los subsidios a la exportación para 2013, pero, dado que no ha habido resultados en la negociación, esta medida no va a implementarse inmediatamente.

Si en el pasado el acuerdo de los dos grandes actores desarrollados fue clave, hoy en día los autores coinciden en señalar que, debido a la ampliación de miembros de la OMC y al ascenso en poder de los emergentes, la UE ya no es un actor determinante para el acuerdo

\footnotetext{
${ }^{36}$ Véase al respecto: Meunier, Sophie (2007): Trading Voices: The European Union in international commercial negotiations, Woodstock, Princeton University Press.

${ }^{37}$ Daugbjerg, Carsten y Swinbank, Alan (2009): Ideas, Institutions and Trade. The WTO and the Curious Role of EU Farm Policy in Trade Liberalization, Oxford, Oxford University Press.

${ }^{38}$ Sobre esta cuestión: McMahon, Joseph A. (2011): The Negotiations for a New Agreement on Agriculture, Leiden, M. Nijhoff.

${ }^{39}$ Para un análisis del estado actual de la negociación de Doha, véase: Orden, David; Blandford, David; Josling, Tim y Brink, Lars: "WTO Disciplines on Agricultural Support. Experience to Date and Assessment of Doha Proposals", International Food Policy Research Institute (May 2011).

${ }^{40}$ Allen, David y Smith, Michael: "Relations with the Rest of the World", Journal of Common Market Studies, vol. 50, (Annual Review 2012), pp. 162-177, esp. la p. 166.
} 
final $^{41}$. Igualmente, dadas las diferentes posiciones de negociación y el inmovilismo de los principales actores se coincide en que es altamente improbable que se llegue a un éxito en esta nueva ronda negociadora y que la liberalización del comercio internacional en el futuro se desarrollará más en el plano bilateral e interregional.

Los otros foros multilaterales de relevancia que ha tratado la cuestión de la seguridad alimentaria son el G-20, donde la UE tiene una representación agregada al igual que algunos de sus Estados miembros y el G-8, donde están los cuatro más desarrollados, pero no la UE ${ }^{42}$. Al ser el foro de referencia para la cooperación internacional en materia económica, la seguridad alimentaria entró en la agenda de los Gs en 2008 como consecuencia de la subida generalizada del precio de los alimentos ${ }^{43}$.

En junio de 2011 los Ministros de Agricultura del G-20 aprobaron un Plan de Acción sobre Volatilidad de los Precios de los Alimentos y Agricultura, a iniciativa de la Presidencia francesa y la Unión Europea, que sería después endosado por los Jefes de Estado y de Gobierno $^{44}$. Este Plan recoge una serie de compromisos que la UE, y los otros miembros, deben cumplir como cooperar para reforzar la productividad agrícola en los países menos avanzados, incrementar la transparencia trasladando datos al Sistema de Información Agrícola de la FAO, intentar alcanzar un acuerdo internacional para eliminar las restricciones y tasas al comercio de alimentos con fines humanitarios, participar en proyectos internacionales de gestión de riesgos del Programa Mundial de Alimentos y avanzar en la regulación de los mercados financieros para poner coto a la especulación que repercute en la volatilidad de precios.

\subsection{Políticas comerciales preferenciales centradas en los alimentos}

Los países en vías de desarrollo son, con carácter general, los más afectados por los desafíos relacionados con la seguridad alimentaria. Muchos de ellos se enfrentan al problema del hambre además del de la pobreza y tienen, por tanto, que formular una estrategia de seguridad alimentaria que encuentre un equilibrio entre producción nacional para consumo interno y exportaciones para obtener divisas y competitividad económica internacional. Esto, a veces, no es fácil por los problemas de falta de institucionalidad que suelen acompañar al poco desarrollo económico.

\footnotetext{
${ }^{41}$ García-Durán Huet, Patricia y Millet, Montserrat (2012): "La UE en el ámbito agrícola. De Uruguay a Doha: una misma estrategia con resultados muy diferentes, en: Barbé Izuel, Esther: Cambio Mundial y Gobernanza Global. La interacción entre la Unión Europea y las instituciones internacionales, Madrid, Tecnos, pp. 86-104; McGuire, Steven M. y Lindeque, Johan P.: "The Diminishing Returns to Trade Policy in the European Union", Journal of Common Market Studies, vol. 48, no 5 (2010), pp. 1329-1349; Meunier, Sophie: "Managing Globalization? The EU in International Trade Negotiations", Journal of Common Market Studies, vol. 45, $\mathrm{n}^{\circ} 4$ (2007), pp. 905-926.

${ }^{42}$ El G-20 es un foro de diálogo sobre gobernanza global, que viene a reemplazar al G-8 incorporando a las economías emergentes. La UE es miembro de pleno derecho junto con los siguientes Estados miembros: Alemania, Reino Unido, Francia e Italia, y Países Bajos y España con estatuto de invitado. El Primer Ministro británico Cameron elaboró y presentó un informe sobre el papel que este foro está llamado a representar en la gobernanza global, véase: Cameron, David: "Governance for Growth. Building Consensus for the Future", Prime Minister of the United Kingdom, Prime Minister's Office, London, (2011).

${ }^{43}$ Así el G-8 en su reunión de 2008 adoptó la Iniciativa de Seguridad Alimentaria de L'Aquila, que suponía el compromiso de los más desarrollados en incrementar su cooperación en materia de alimentos con aquellos países que estaban sufriendo los efectos directos de la crisis.

${ }^{44}$ Véase respectivamente: "Ministerial Declaration Action Plan on Food Price Volatility and Agriculture", Meeting of G-20 Agriculture Ministers, Paris, (22 y 23 Junio 2011); G-20 Leaders' Summit, Communiqué, Cannes, (3-4 Noviembre 2011), ptos. 18 y 19.
} 
En la actual estructura económica internacional coincide además que muchos países en vías de desarrollo son además exportadores de productos agrícolas no elaborados. Es, por ello una relevante herramienta de cooperación internacional el acceso preferencial al mercado de los países desarrollados. La UE ha aplicado un modelo de acceso preferencial para los productos procedentes de países en vías de desarrollo: el Sistema de Preferencias Generalizadas (SPG) y el Sistema de Preferencias Generalizadas Plus (SPG+). Ambos sistemas prevén para los países terceros en vías de desarrollo ventajas arancelarias para determinados productos agrícolas como forma de cooperación. Ambos responden a una cierta condicionalidad democrática, siendo el Plus más exigente en términos de democracia y buen gobierno. Análisis empíricos sobre los efectos que el sistema SPG ha tenido para las exportaciones de los productos agrícolas de los países en vías de desarrollo hacia la UE ofrecen resultados globalmente positivos ${ }^{45}$.

Además de ese sistema generalizado, la UE utiliza la vía de los acuerdos comerciales dentro de sus relaciones preferenciales bilaterales o multilaterales. Así, marcos de relación como el ACP, la Asociación Euro-Mediterránea o políticas bilaterales tienen como núcleo un acceso preferencial a los mercados europeos para los productos agrícolas procedentes de estos países. Entre la gran cantidad de los existentes, puede citarse como ejemplo que en el marco de la Vecindad Sur se ha firmado en febrero de 2012 con Marruecos un acuerdo comercial preferencial que supone un importante avance hacia el libre comercio ${ }^{46}$. Elimina o reduce en un 55\% las tasas para los productos agrícolas y de la pesca marroquíes y reduce en un $70 \%$ las tarifas para los productos agrícolas y procedentes de la pesca de la UE de una forma progresiva para los próximos diez años.

\subsection{La acción a través de la cooperación para el desarrollo}

Finalmente, resulta relevante examinar la cooperación para el desarrollo, en sentido estricto, de la UE en materia de seguridad alimentaria, ya que este organismo, como agregado, es el primer donante a escala global y la Comisión Europea aisladamente el segundo en términos absolutos.

En primer lugar, es preciso tener en cuenta la dimensión bilateral de esa cooperación, esto es, la contribución de la Ayuda Oficial al Desarrollo Europea a reforzar específicamente la seguridad alimentaria de terceros países o grupos de países. En los distintos instrumentos de la cooperación europea, vemos que la seguridad alimentaria es un ámbito de actuación tanto en la cooperación ACP, como en el Instrumento de Desarrollo y en el Instrumento Europeo de Vecindad y Asociación ${ }^{47}$. Es, un objetivo, por tanto, en el caso de la cooperación con todos los países en vías de desarrollo que no tengan una perspectiva de futura adhesión a la UE. Esa cooperación se materializa a través del diálogo bilateral entre las instituciones

\footnotetext{
${ }^{45}$ Véase: Aiello, Francesco y DeMaria, Federica: "Do trade preferential agreements enhance the exports of developing countries?: Evidence from the EU GSP", Economía Internazionale, vol. 65, no 3 (2012), pp. $371-404$.

${ }^{46}$ Este acuerdo, firmado en febrero de 2012, entró en vigor el 1 de mayo del mismo año, tras ser aprobado por el Parlamento Europeo.

${ }^{47}$ Véanse respectivamente: Acuerdo Interno entre los Representantes de los Gobiernos de los Estados miembros, reunidos en el seno del Consejo, relativo a la financiación de la ayuda comunitaria concedida con cargo al marco financiero plurianual para el periodo 2008-2013 de conformidad con el Acuerdo de Asociación ACP-CE y a la asignación de ayuda financiera a los países y territorios de ultramar a los que se aplica la parte cuarta del Tratado CE, DO L 247, de 9.9.2006, p. 32; Reglamento CE nº 1905/2006, del Parlamento Europeo y del Consejo, de 18 de diciembre de 2006, por el que se establece un Instrumento de Financiación de la Cooperación al Desarrollo, DO L 378, de 27.12.2006, p. 41; Reglamento CE n 1638/2006, del Parlamento Europeo y del Consejo, de 24 de octubre de 2006, por el que se establecen las disposiciones generales relativas a la creación de un Instrumento Europeo de Vecindad y Asociación, DO L 310, de 9.11.2006, p. 1.
} 
europeas y el gobierno del país en cuestión, donde se consensuan los objetivos de la cooperación, las áreas prioritarias así como las formas de sostenimiento comunitario y que se plasman en los Documentos Estratégicos y los Documentos Indicativos Nacionales.

En el ámbito de la Vecindad Sur, además de la cooperación bilateral, se ha aprobado en 2012 un programa multilateral denominado ENPARD (Programa de la Vecindad Europea para la Agricultura y el Desarrollo Rural) con el objetivo directo de mejorar la seguridad alimentaria de estos países ${ }^{48}$. Pretende ayudar a modernizar el sector agrícola de los países socios elevando la productividad, mejorando la calidad de la producción y el acceso al mercado. Busca que los países terceros se beneficien del comercio global y establezcan las bases para un desarrollo rural sostenible y un nivel básico de seguridad alimentaria y protección contra la volatilidad de los precios.

Además de la cooperación país por país, la UE también ha desarrollado un enfoque horizontal para abordar actuaciones relacionadas con determinados problemas transversales en cualquier país del mundo o a través de la cooperación con otras organizaciones o instituciones internacionales. En este ámbito existe un programa concreto destinado a abordar la "Seguridad Alimentaria" dotado con 1.709 millones de euros para el periodo 2007-2013". El objetivo de este programa es aumentar la seguridad alimentaria a favor de la población más pobre y vulnerable, contribuyendo a la consecución del Objetivo de Desarrollo del Milenio relativo a la pobreza y el hambre.

El Reglamento prevé financiación para proyectos de ONGs y programas internacionales en los siguientes ámbitos y actuaciones: a) contribuir a la provisión de bienes públicos internacionales; b) apoyar programas mundiales, continentales y regionales para formular políticas agrícolas, promover estrategias nacionales de seguridad alimentaria y reducción pobreza, desarrollar el programa mundial de seguridad alimentaria y crear sistemas de información y alerta rápida; c) defender y hacer avanzar el programa de la seguridad alimentaria en el debate internacional; d) tratar la inseguridad alimentaria en situaciones excepcionales de transición y fragilidad de estado; y e) impulsar políticas, estrategias y planteamientos innovadores en seguridad alimentaria. A través de este programa horizontal, por ejemplo, la UE materializa su aportación al Programa Mundial de Alimentos de las Naciones Unidas.

Además, en diciembre de 2008, como consecuencia del compromiso del G-8 de L'Aquila, la UE lanzó la llamada Food Facility para abordar las consecuencias de la crisis alimentaria de $2008^{50}$. Esta iniciativa, programada para tres años y con una dotación de 1.000 millones de euros, pretendía dar respuesta rápida a las consecuencias de la subida de precios en alimentos para los países en vías de desarrollo, siendo un instrumento que está a medio camino entre la ayuda de emergencia y la cooperación a largo plazo. Sus objetivos son animar a los agricultores a que incrementen la oferta; tratar los efectos de la volatilidad de los precios sobre las poblaciones locales; e incrementar la capacidad productiva y mejorar la gestión agrícola en el largo plazo. Financia medidas para mejorar el acceso a los insumos y servicios

\footnotetext{
48 "New Commission Aproach on Agriculture in Neighbourhood Launched", Press Release, European Commission, Brussels (31 Mayo 2012), en http://ec.europa.eu/europeaid/news/documents/enpard_with_fule quote_en.pdf.

${ }^{49}$ Véase el artículo 15 del Reglamento CE n ${ }^{\circ}$ 1905/2006, ya citado.

${ }^{50}$ Reglamento (CE) n ${ }^{\circ} 1337 / 2008$ del Parlamento Europeo y del Consejo, de 16 de diciembre de 2008, por el que se establece un mecanismo de respuesta rápida frente a la fuerte subida de los precios de los productos alimenticios en los países en desarrollo, DO L 354, 31.12.2008, p. 62.
} 
agrícolas, redes de seguridad para mantener la producción agrícola, medidas a escala local para incrementar la producción como microcréditos, inversiones, equipos, almacenamiento...

La seguridad alimentaria global y las de terceros países, por tanto, se ve afectada no sólo por el modelo de seguridad alimentaria europea y la PAC, sino también por la acción exterior europea tanto en cuestiones de comercio como de cooperación para el desarrollo garantizando la seguridad alimentaria de terceros. Estos dos ámbitos no siempre son compatibles: las medidas PAC no siempre son coherentes con el objetivo de garantizar la seguridad alimentaria de los países en vías de desarrollo. La única preocupación de la seguridad alimentaria de terceros tampoco puede guiar todas las decisiones políticas para establecer un modelo de seguridad alimentaria europea.

\section{Conclusiones}

La UE uno de los principales actores de la seguridad alimentaria a escala mundial, no sólo por la importancia de su producción sino también por ser uno de los principales mercados de alimentos que reciben importaciones de terceros países y ser actor comercial y de la cooperación relevante. De ahí la importancia que las políticas europeas de seguridad alimentaria, la PAC, tienen en la formulación de las estrategias políticas en la materia para el resto de los países del mundo.

El modelo europeo de seguridad alimentaria, ha evolucionado desde un modelo productivista a otro multifuncional, abierto a la integración progresiva en los mercados internacionales. La próxima reforma planteada desde la Comisión sigue estas pautas, priorizando bienes públicos como la calidad del medio ambiente, la seguridad de los consumidores y la contribución de los agricultores al modelo social sobre la producción de alimentos y la autosuficiencia europea, que ha ido disminuyendo progresivamente en importancia a lo largo del tiempo.

Si examinamos su acción exterior, podemos ver que la UE ha promovido la liberalización del comercio sobre alimentos sólo limitadamente ya que han primado los intereses internos de protección de sus agricultores. La UE es también el principal socio de los países en vías de desarrollo, a través de concesiones comerciales preferenciales y cooperación bilateral y multilateral. Existe un amplio clamor sobre la necesidad de lograr la coherencia entre la PAC y el desarrollo, no siempre atendida, aunque se están obteniendo logros parciales.

Finalmente podemos preguntar si es inteligente y viable mantener el modelo multifuncional para la seguridad alimentaria europea a largo plazo. Los analistas llaman la atención sobre que para 2050 será necesario doblar la producción mundial de alimentos por el incremento de la población y el cambio de dieta derivado del acceso al desarrollo de los emergentes. Además hay que tener en cuenta los rendimientos decrecientes de la actividad agrícola derivados de las consecuencias del cambio climático ${ }^{51}$. No es ni posible ni lógico volver al modelo de los años 60 pero quizás habría que plantearse una PAC que volviera, gradualmente, a incentivar la producción a la vez que tomara en consideración otros objetivos.

\footnotetext{
${ }^{51}$ Un ejemplo del debate académico existente sobre los diferentes modelos de política agrícola es: Almås, Reidar y Campbell, Hugo (2012): Rethinking Agricultural Policy Regimes: Food Security, Climate Change and the Future Resilience of Global Agriculture, Bingley, Emerald.
} 
Esto puede verse desde una perspectiva y una inquietud de seguridad alimentaria pero también parece aconsejable para cumplir el objetivo del Tratado de seguir garantizando a los consumidores precios razonables. Igualmente no podemos dejar de advertir que, en un contexto internacional de demanda creciente de alimentos, la productividad de la agricultura y la industria alimentaria europea puede ser un sector relevante para la competitividad de la economía europea perseguida por la Estrategia Europa 2020. Quizás sería razonable explorar las posibilidades de un modelo que podríamos calificar de neoproductivista, que incentivara la producción como en los inicios, pero de una manera controlada, combinándola con otros requerimientos como la dimensión medioambiental, la calidad y la protección de la seguridad de los consumidores. 